\title{
Avaliação dos serviços odontológicos na Atenção Básica na Região do Baixo Amazonas - Pará
}

\author{
Evaluation of odontological services in the basic attention in the Region of Baixo \\ Amazonas - Pará
}

Marizeli Viana de Aragão Araújo ${ }^{1}$

Márcia de Oliveira Correa ${ }^{2}$

Mayara Sabrina Luz Miranda ${ }^{3}$

Helder Henrique Costa Pinheiro ${ }^{1}$

Danielle Tupinambá Emmi ${ }^{1}$

Regina Fátima Feio Barroso ${ }^{1}$

\begin{abstract}
RESUMO
A avaliação da qualidade dos serviços de saúde é uma ferramenta importante na qualificação de ações e planejamento para promover a melhoria do acesso e da qualidade das ações de Atenção Básica em Saúde. Com o objetivo de permitir a ampliação do acesso e melhoria da qualidade de Atenção Básica no Brasil foi instituído em 2011 o Programa Nacional para Melhoria do Acesso e da Qualidade da Atenção Básica (PMAQ-AB). A proposição desse estudo foi avaliar os serviços odontológicos na Atenção Básica em nove municípios da região do Baixo Amazonas no estado do Pará. Trata-se de um estudo descritivo realizado a partir de dados secundários do primeiro ciclo do PMAQ-AB onde foram avaliadas as seguintes variáveis: visita domiciliar; biópsia e câncer de boca; procedimentos clínicos e atendimentos às gestantes e escolares. Os resultados encontrados demonstraram que os procedimentos clínicos, atendimentos às gestantes e escolares eram satisfatórios, porém foi observada deficiência nos procedimentos relacionados ao câncer bucal. Diante desses resultados, pode-se sugerir que as equipes de saúde bucal estavam preparadas para atender a maioria dos serviços odontológicos, porém apresentavam dificuldades em executar os que envolviam o câncer bucal, o que pode refletir uma deficiência na infraestrutura dos municípios para a execução destes serviços e/ou na formação inadequada do cirurgião dentista.
\end{abstract}

Palavras chave: Odontologia. Atenção Primaria à Saúde. Avaliação de Serviços de Saúde. Qualidade da Assistência à Saúde.

\begin{abstract}
The evaluation of the quality of health services is an important tool in the qualification of actions and planning to promote the improvement of access and quality of actions of Basic Health Care. The National Program for the Improvement of Access and Quality of Primary Care (PMAQ-AB) was established in 2011 with the objective of increasing access to and improving the quality of basic care in Brazil. The purpose of this study was to evaluate the dental services in Basic Care in nine municipalities in the Baixo Amazonas region in the state of Pará. This is a descriptive study based on secondary data from the first cycle of PMAQ$\mathrm{AB}$ where the following were evaluated: variables: home visit; biopsy and cancer of the mouth; clinical procedures and care for pregnant women and schoolchildren. The results showed that the clinical procedures, care for pregnant women and schoolchildren were satisfactory, but a deficiency in oral cancer procedures was observed. In view of these results, it can be suggested that the oral health teams were prepared to attend most dental services, but they had difficulties in performing those involving oral cancer, which may reflect a deficiency in the municipalities infrastructure for the execution of these services and / or inadequate training of the dentists.
\end{abstract}

Keyword: Dentistry. Primary Health Care. Service Rating. Quality Health Care.

\footnotetext{
${ }^{1}$ Professor (a) Doutor, Faculdade de Odontologia, Universidade Federal do Pará

${ }^{2}$ Especialista em Ortodontia, Faculdade de Medicina e Odontologia São Leopoldo Mandic

${ }^{3}$ Mestre, Universidade Federal do Pará
} 


\section{INTRODUÇÃo}

Historicamente, a ideia de atenção primária foi utilizada como forma de organização dos sistemas de saúde pela primeira vez em 1920 no chamado Relatório Dawson, contrapondo ao modelo flexineriano americano de cunho curativo, fundado no reducionismo biológico e na atenção individual. O referido relatório organizava o modelo de atenção em centros de saúde primários e secundários, serviços domiciliares, serviços suplementares e hospitais de ensino. Os centros de saúde primários e os serviços domiciliares deveriam estar organizados de forma regionalizada, onde a maior parte dos problemas de saúde deveria ser resolvida ${ }^{1}$.

A Atenção Básica à Saúde (ABS), também chamada de Atenção Primaria à Saúde, foi reformulada de acordo com os preceitos do novo modelo de saúde vigente, o Sistema Único de Saúde (SUS). Passa a ter como uma de suas características um nível de saúde com ações no âmbito individual e coletivo, que abrange a promoção, a proteção, a prevenção de agravos, o diagnóstico, o tratamento, a reabilitação, a redução de danos e a manutenção da saúde ${ }^{2}$.

Nas duas últimas décadas, a Atenção Básica à Saúde passou por mudanças importantes. Em 1994 o Ministério da Saúde lançou o Programa Saúde da Família (PSF), hoje denominada Estratégia Saúde da Família (ESF), no âmbito da ABS, inspirado em experiências vividas por outros países, como Inglaterra, Cuba e Canadá, cuja saúde pública tinha qualidade alcançada por meio da promoção da saúde e prevenção dos agravos em saúde 3 .

A justificativa para implantação do PSF na Atenção Básica foi a necessidade de substituir o modelo assistencial, centrado na doença e no cuidado individual por um modelo relacionado com os princípios da Universalidade, Equidade e Integralidade de atenção preconizado pelo SUS. Além disso, teve como princípio básico, o trabalho em equipe multiprofissional, com território definido e adstrição da população a ser trabalhada por cada equipe de saúde. O programa prioriza ainda a promoção e proteção à saúde da família e dos indivíduos doentes ou livres de doenças, de forma integral e contínua ${ }^{4}$.

No que diz respeito à saúde bucal, pode-se dizer que a inserção dos serviços odontológicos nos moldes da ESF se deu de forma paralela e afastada do processo de organização dos demais serviços de saúde. A partir da inserção da Equipe de Saúde Bucal (ESB) na ESF em 2002, há uma tendência de reorganização e inserção da prática odontológica na Atenção Básica ${ }^{5}$.

O primeiro Levantamento Epidemiológico em Saúde Bucal realizado pelo Ministério da Saúde (MS) em 1986, com grupo de pessoas de 50-59 anos, mostrou índice $\mathrm{CPOD}$, que indica o número de dentes permanentes cariados, perdidos (extraídos e com extração indicada) e restaurados, de 27,2 para essa faixa etária, com $86 \%$ de participação dos dentes extraídos, já sugerindo as péssimas condições em que se encontravam as pessoas com mais de sessenta anos ${ }^{6}$. O elevado número de dentes extraídos encontrado neste levantamento, evidenciou um serviço odontológico ineficaz e que precisava ser reformulado para suprir as necessidades e melhorar a saúde bucal de toda a população.

Assim, a Política Nacional de Saúde Bucal propôs a incorporação progressiva de ações de promoção e proteção em saúde, como fluoretação das águas de abastecimento, educação em saúde coletiva e individual, higiene bucal supervisionada e aplicações tópicas de flúor?

Inúmeros esforços têm sido empreendidos com o objetivo de adequar as estratégias previstas na Política Nacional de Atenção Básica (PNAB), para reconhecer a qualidade dos serviços de $\mathrm{ABS}$ ofertados à sociedade, $\mathrm{e}$ estimular a ampliação do acesso. Nesse sentido, o Ministério da Saúde lançou o Programa Nacional de Melhoria do Acesso e da Qualidade da Atenção Básica (PMAQ-AB).

O PMAQ-AB foi instituído pela Portaria $\mathrm{n}^{\circ} 1.654$ GM/MS, de 19 de julho de 2011 com o objetivo de permitir a ampliação do acesso e melhoria da qualidade da Atenção Básica em todo o Brasil. O Programa está estruturado em quatro fases que conformam um ciclo contínuo: fase 1 - adesão e contratualização; fase 2 desenvolvimento; fase 3 - avaliação externa; e fase 4 recontratualização ${ }^{8}$.

A mesorregião do Baixo Amazonas, localizada no oeste do estado do Pará compreende uma superfície de aproximadamente $352.613 \mathrm{~km}^{2}$, superior às áreas do Reino Unido e da Coreia do Sul somadas, e abriga uma população estimada de 784.389 mil habitantes, 
dividida em quinze municípios: Almerim, Porto de Moz, Faro, Juruti, Óbidos, Oriximiná, Terra Santa, Alenquer, Belterra, Curuá, Mojuí dos Campos, Monte Alegre, Placas, Prainha e Santarém ${ }^{9}$.

Desta forma, a utilização da avaliação da qualidade passou a se constituir numa ferramenta importante na qualificação das ações e planejamentos para promover a melhoria do acesso e da qualidade da Atenção Básica. Assim, este estudo tem como objetivo avaliar a qualidade dos serviços odontológicos oferecidos na Atenção Básica na Mesorregião do Baixo Amazonas no estado do Pará.

\section{Metodologia}

Trata-se de um estudo descritivo e analítico, cujos dados foram provenientes da avaliação externa do primeiro ciclo (Fase 3) do PMAQ-AB realizado entre os anos de 2012 e 2013 no Pará, no qual foram coletadas informações para a análise das condições de acesso e de qualidade das ESB participantes do Programa. Para a realização da avaliação externa foi utilizado o instrumento de avaliação elaborado pelo Ministério da Saúde e aplicado por avaliadores selecionados pela Universidade Federal do Pará (UFPA), responsável pelo Programa no estado.

O instrumento avaliativo estava organizado em três módulos, Módulo I - Observação na Unidade Básica de Saúde; Módulo II - Entrevista com o profissional da equipe e verificação de documentos na UBS, direcionado para a realização da avaliação externa das referidas equipes; Módulo III - Entrevista com o usuário na Unidade Básica de Saúde. Para o presente artigo foram analisadas as seguintes variáveis: visita domiciliar; biópsia e câncer de boca; procedimentos clínicos e atendimentos às gestantes e escolares, cujos dados estavam disponíveis no Módulo II.

Como a fase inicial do PMAQ compreende a adesão de municípios ao programa, dos 15 municípios que fazem parte da região do Baixo Amazonas, 06 não realizaram a contratualização de suas Equipes de Saúde Bucal ao PMAQ-AB. Dessa forma, os dados analisados foram os coletados em 09 municípios da região. Após a coleta, os dados foram enviados ao Ministério da Saúde, validados e disponibilizados às instituições parceiras ${ }^{10}$.

Esta pesquisa foi autorizada pelo Comitê de Ética em Pesquisa da Universidade Federal do Rio Grande do Sul sob o número 21.904, tratando-se de projeto multicêntrico, onde a UFPA está inserida.

Os dados coletados foram apurados em banco de dados no formato de planilha eletrônica, foram transferidos para o software Statistical Package for the Social Sciences (SPSS 20.0), onde foram realizados os cálculos das frequências das variáveis observadas.

\section{Resultados}

Na mesorregião do Baixo Amazonas, dos nove municípios que aderiram ao PMAQ- AB, 36 Equipes de Saúde Bucal foram contratualizadas. A Tabela 1 expressa os dados relativos à visita domiciliar. De acordo com os dados obtidos, observou-se que das 36 equipes, apenas $21(68,3 \%)$ faziam a visita.

Com relação às 21 equipes que realizavam a visita domiciliar, apenas $04(11,1 \%)$ faziam uso de documentos com definição de situações prioritárias para visita. Quanto à realização de consultas para idoso/doença crônica-domiciliado/acamado, 16 (44,4\%) faziam esse tipo de consulta. Procedimentos clínicos no cuidado domiciliar eram efetuados somente por 09 equipes $(25,0 \%)$.

Os dados referentes à realização do exame da biópsia e câncer bucal estão representados na Tabela 2. Observase que apenas $04(11,1 \%)$ equipes realizavam a coleta do material para o exame, sendo que dos Cirurgiões Dentistas (CD) que coletavam o material, apenas 02 $(5,6 \%)$ realizavam no mesmo momento que ocorria a suspeita da doença. Foram relatados 03 casos $(8,3 \%)$ confirmados de câncer bucal.

Outro dado importante é o prazo de entrega do laudo pelo patologista/laboratório. Segundo a pesquisa, em metade dos casos enviados para análise, o resultado demorou em média de 30 a 60 dias. 
A Tabela 3 representa os procedimentos considerados básicos da odontologia que eram realizados na sua grande maioria por quase $100 \%$ das ESB, com exceção dos casos de remoção de cisto e frenectomia que eram realizados por menos da metade das ESB.

O acompanhamento das gestantes e ações direcionados aos pré-escolares realizadas pelas ESB estão representados na Tabela 4.

O acompanhamento das gestantes era realizado por $27(75,0 \%)$ das ESB.

Em relação à assistência aos escolares, 31 (86,1\%) das ESB realizavam atendimento em crianças de até 5 anos de idade. Vinte e seis $(72,2 \%)$ ESB realizavam atividades em creche ou escolas, contudo somente 19 $(52,8 \%)$ faziam registro das atividades. O levantamento do número de crianças que necessitavam de tratamento era feito por 36,1\% (13) ESB, percentual muito aquém do desejado.

\section{DisCuSSÃo}

Lorena-Sobrinho et $\mathrm{al}^{11}$ ao avaliarem o cuidado domiciliar executado pelas ESB em Pernambuco verificaram que este atendimento era executado pelo $\mathrm{CD}$ em 589 equipes $(73,53 \%)$ e pelo técnico ou auxiliar em saúde bucal em 496 (61,92\%). Percentual bem superior ao observado no presente estudo, o que demonstra a necessidade de intensificar essa prática no estado do Pará, já que a visita domiciliar é um recurso útil para a promoção de saúde bucal e um dos pilares da ESF, uma vez que, a presença do profissional in loco traz benefícios para a compreensão da realidade do usuário, ajudando assim no processo saúde-doença, além de estabelecer e fortalecer o vínculo quando há problemas de adesão ao tratamento.

De acordo com a PNAB, a visita domiciliar é recomendada nos casos em que o usuário possua problemas de saúde controlados/compensados e com dificuldade ou impossibilidade física de locomoção até uma unidade de saúde ${ }^{12}$. Ações curativas também podem ser utilizadas para intervenções em pacientes impossibilitados de se deslocarem até à Unidade Saúde da Família, considerando-se, neste caso, a disponibilidade de equipamentos adequados ${ }^{13}$.

O baixo índice de visitas encontrado neste estudo pode refletir o perfil do cirurgião dentista da equipe, que não teria a formação adequada para atuar na ESF, assim como, inexistência de condições para a realização do atendimento domiciliar.

No que diz respeito ao câncer bucal, Costa Júnior e Serra ${ }^{14}$ em estudo realizado no município de Resende (RJ), identificaram que $90 \%$ dos CD das ESB não haviam recebido capacitação institucional sobre diagnóstico precoce e encaminhamento de pacientes com suspeita de lesões orais consideradas précancerígenas, justificando as limitações destes profissionais.

$\mathrm{O}$ atraso no diagnóstico, e consequentemente, o atraso no tratamento pode comprometer o prognóstico e a sobrevida do indivíduo doente.

Foram relatados 03 casos $(8,3 \%)$ confirmados de câncer bucal. Essa baixa incidência pode estar relacionada ao fato de que apenas 04 profissionais na região realizavam a coleta do material da biópsia para o diagnóstico da doença. As regiões Norte, Nordeste e Centro Oeste apresentam um desempenho crítico quanto ao acompanhamento de casos suspeitos ou confirmados de câncer de boca. $\mathrm{O}$ acesso para a especialidade de estomatologia na região Norte ainda é restrito. O tempo de espera é de até 30 dias e só atende a cerca de $30 \%$ dos $\operatorname{casos}^{15}$.

No estado do Pará a oferta de serviços não supre a demanda. A quantidade de Centros de Especialidades Odontológicas (CEO) no estado é de 33 e no Baixo Amazonas apenas 05. De acordo com Lombardo et al. ${ }^{16}$ há municípios que apresentam falha na rede de atenção por não possuírem centros de referência para encaminhamento. Muitos casos suspeitos de câncer são diagnosticados na capital (Belém), normalmente nos serviços de referência das instituições de ensino superior. Dessa maneira, o acesso ao diagnóstico oral é demorado e inacessível a muitos usuários. Segundo Casotti et al. ${ }^{15}$, na região Norte a estomatologia é a especialidade que apresenta maior espera para atendimento $(63,8 \%)$, no intervalo de um ano. 
Quando questionados sobre ações de prevenção, 23 $(63,9 \%)$ das equipes afirmaram realizar essas ações, números próximos ao estudo realizado no estado de Pernambuco em 2012, onde 58,92\% das ESB realizavam esse tipo de serviço ${ }^{11}$. As ações de prevenção são necessárias, já que por meio destas, os profissionais orientam sobre a importância do autoexame bucal para o diagnóstico precoce e com relação aos fatores de risco que contribuem para o aparecimento da doença, além de serem de baixo custo e abrangerem um grande número de pessoas.

A implementação de programas de rastreamento ou prevenção em nível regional ou nacional, aliados a programas de educação permanente e continuada para os profissionais de saúde em geral e da Odontologia, são medidas importantes para reduzir o atraso no diagnóstico, conjuntamente com a realização de exames clínicos periódicos ${ }^{16}$.

Diante das informações obtidas, observou-se que os atendimentos clínicos mais corriqueiros são disponibilizados à população, justificando o fato de que, é na Atenção Básica que são solucionados os problemas de saúde bucais mais frequentes. Por mais que as ações da ESF sejam destinadas às práticas coletivas, devido à histórica exclusão por serviços clínicos e à grande demanda reprimida, as Diretrizes da Política Nacional de Saúde Bucal sugerem que de 75 a $85 \%$ das horas trabalhadas pelo cirurgião dentista na ESF sejam utilizadas na assistência curativa ${ }^{17}$.

Os resultados relacionados às gestantes podem ser considerados expressivos e importantes, pois durante a gravidez ocorrem transformações biológicas e psicológicas que podem predispor as grávidas à situações de risco à saúde bucal, ocasionadas pelas alterações hormonais, as quais podem intensificar processos inflamatórios periodontais ou o surgimento de novas patologias na cavidade bucal.

Geralmente durante a gravidez o acesso à assistência odontológica é comprometido, pois há usuárias que acreditam que o tratamento odontológico é contraindicado, visto que qualquer procedimento odontológico implicaria em riscos à saúde do bebê. Por outro lado, há profissionais que, por desconhecimento ou insegurança, adiam procedimentos ou não intervêm de modo resolutivo visando uma saúde bucal satisfatória da gestante ${ }^{18}$.

Estudo realizado por Esposti et al. ${ }^{19}$ relata que gestantes entrevistadas na grande Vitória, estado do Espírito Santo, ressaltaram a indisponibilidade de cuidado odontológico de mulheres que gostariam de ter acesso a esse serviço. Além disso, outro fator observado pelas gestantes entrevistadas diz respeito a grande distância existente entre a residência e o serviço disponível, o que provocava o deslocamento entre bairros e municípios vizinhos, em áreas descobertas pela ESF, interferindo na continuidade do cuidado pré-natal, resultando no descumprimento do mínimo de consultas previstas, diferentemente do que acontecia nas áreas cobertas pela ESF.

As visitas domiciliares devem efetuar a busca ativa de gestantes, auxiliando sua inserção no processo de cuidado pré-natal. A Política Nacional de Saúde Bucal visa à ampliação da assistência odontológica às gestantes que necessitam de prioridade nos cuidados e apresentam quadros de saúde bucal peculiares ${ }^{19}$.

Villardi e Cyrino $^{20}$ realizaram um estudo com profissionais da ESF de Botucatu-SP e ressaltaram que a atenção à saúde da criança em idade escolar requer a capacitação dos profissionais para a compreensão das necessidades da criança, incentivando o trabalho multiprofissional e evitando as práticas fragmentadas. Além disso, sugerem a organização de diálogos com as escolas para que se possa valorizar e viabilizar redes de práticas e saberes. Estruturar e valorizar práticas de saúde voltadas às crianças em idade escolar é essencial na busca de caminhos que qualifiquem a saúde dos pequenos.

Sabe-se que quanto mais precocemente comportamentos saudáveis relacionados à saúde bucal forem inseridos no contexto da criança, maior será a probabilidade na manutenção da estabilização da saúde ao longo dos anos, já que as crianças possuem grande capacidade de imitar ações que presenciem e apresentam facilidade em alterar hábitos errôneos ${ }^{21}$ 


\section{Conclusão}

A presente pesquisa demonstrou que nos municípios da mesorregião do Baixo Amazonas que aderiram ao PMAQ, as ESB realizavam atendimentos na grande maioria dos procedimentos expostos, porém no que se referia a biópsia para diagnóstico do câncer bucal, as equipes precisavam se qualificar para realizar diagnóstico precoce e encaminhar para a realização do tratamento adequado, pois apenas $11,1 \%$ realizava esse tipo de procedimento.

Os resultados encontrados revelaram que os procedimentos referentes aos serviços de atenção básica são ofertados à população do Baixo Amazonas, porém estudos mais aprofundados são importantes para identificar se os serviços ofertados correspondem à demanda daquela região.

\section{REFERÊNCIAS}

1. Matta GC, Morosini MV. Dicionário da Educação Profissional em Saúde, Rio de Janeiro, 2009. Disponível em:<http://www.epsjv.fiocruz.br/upload/d/Atencao_Pri maria_a_Saude_-_recortado.pdf $>$. Acessado em: 08 abr. 2016.

2. Brasil. Ministério da Saúde. Portaria GM $n^{\circ}$ 648, de 28 de março de 2006. Aprova a Política Nacional de Atenção Básica, estabelecendo a Revisão de Diretrizes e Normas para a Organização da Atenção Básica para o Programa Saúde da Família (PSF) e o Programa Agentes Comunitários de Saúde (PACS). Brasília, DF, 2006.

3. Brasil. Ministério da Previdência e Assistência Social. Instituto de Assistência Médica da Previdência Social. Resolução $n^{\circ} 258$ de 07 de janeiro de 1991. Norma Operacional Básica $n^{\circ} 1 / 92$. Diário Oficial da União. Brasília, DF, 1991.

4. Scherer MDA, Mariano SRA, Ramos FRS. Rupturas e resoluções no modelo de Atenção à Saúde: reflexões sobre a estratégia saúde da família com base nas categorias kuhnianas. Interface-Comunic., Saúde, Educ., 2004/2005; 9(16):53-66.
5. Brasil. Ministério da Saúde. Secretaria de Atenção à Saúde. Departamento de Atenção Básica. Coordenação Nacional de Saúde Bucal. Caderno de Atenção Básica, $n^{o} 17$ Brasília, DF, 2006.

6. Brasil. Ministério da Saúde. Levantamento Epidemiológico em Saúde Bucal: Brasil, Zona Urbana, 1986. Brasília: Divisão Nacional de Saúde Bucal, Brasília, DF, 1988. Disponível em: <http://bvsms.saude. gov.br/bvs/publicacoes/Sala5545.pdf > Acessado em: 07 abr. 2016.

7. Brasil. Ministério da Saúde. Departamento de Atenção Básica Coordenação Nacional de saúde bucal. Política Nacional de Saúde Bucal. Brasília, DF, 2004. Disponível em: <http://conselho.saude.gov.br/web_comissoes/cisb/ doc/ politica_nacional.pdf $>$. Acessado em: 07 abr. 2016.

8. Brasil. Ministério da Saúde. Departamento de Atenção Básica. Brasil. Programa Nacional de Melhoria do Acesso e da Qualidade da Atenção Básica (PMAQ): manual instrutivo / Ministério da Saúde. Brasília, DF, 2011.

9. Instituto Brasileiro de Geografia e Estatística. Cidades. 2016. Disponível em: https://cidades.ibge.gov.br. Acessado em: 17 out. 2017.

10. Machado JFFP, De Carli AD, Kodjaoglanian VL, Santos MLM. Educação permanente no cotidiano da Atenção Básica no Mato Grosso do Sul. Saúde Debate, 2015; 39(104):102-13.

11. Lorena-Sobrinho JE, Martelli PJL, Albuquerque MSV, Lyra TM, Farias SF. Acesso e qualidade: avaliação das Equipes de Saúde Bucal participantes do PMAQ-AB 2012 em Pernambuco. Saúde Debate, 2015; 39(104):12435.

12. Brasil. Ministério da Saúde. Política Nacional de Atenção Básica. Brasília, DF, Ministério da Saúde, 2012.

13. Faccin D, Sebold R, Carcereri DL. Processo de trabalho em saúde bucal: em busca de diferentes olhares para compreender e transformar a realidade. Ciência \& Saúde Coletiva, 2010; 15(1):1643-52.

14. Costa-Júnior S, Serra CG. Diagnóstico e continuidade do cuidado do câncer bucal em pacientes acompanhados pelas Equipes de Saúde Bucal do Programa de Saúde da Família: a experiência do município de Resende, no Estado do Rio de Janeiro. Cadernos UniFOA, 2011; 15:73-90.

15. Casotti E, Contrato PC, Fonseca ABM, Borges PKO, Baldini MH. Atenção em saúde bucal no Brasil: uma análise a partir da avaliação externa do PMAQ-AB. Saúde Debate, 2014; 38:140-57. 
16. Lombardo EM, Cunha AR, Carrard VC, Bavaresco CS. Atrasos nos encaminhamentos de pacientes com câncer bucal: avaliação qualitativa da percepção dos cirurgiões dentistas. Ciência \& Saúde Coletiva, 2014; 19(4):122332.

17. Brasil. Ministério da Saúde. Secretaria de Atenção à Saúde. Departamento de Atenção Básica. Coordenação Nacional de Saúde Bucal. Diretrizes da Política Nacional de Saúde Bucal. Brasília, DF, 2004.

18. Santos-Neto ET, Oliveira AE, Zanodade E, Leal MC. Acesso à assistência odontológica no acompanhamento pré-natal. Ciência \& Saúde Coletiva, 2012; 17(11):305768.

19. Esposti CDD, Oliveira AE, Santos Neto ET, Travassos C. Representações sociais sobre o acesso e o cuidado prénatal no Sistema Único de Saúde da região metropolitana da grande Vitória, Espírito Santo. Saúde Soc., 2015; 24(3):765-99.

20. Villardi ML, Cyrino EG. O cuidado à criança em idade escolar: percepções de equipes da Estratégia Saúde da Família. Rev Bras Med Fam Comunidade, 2012; 7(24):177-83.

21. Bijella MFTB, Bijella VT, Figueiredo MC. Avaliação de um programa odontológico, com bases educativa, preventiva e curativa, desenvolvido com pré-escolares durante 12 meses. Cecade News, 1995; 3(2):1-5. 


\section{TABelaS}

Tabela 1- Informações gerais sobre a visita domiciliar analisadas na ESB nos municípios do Baixo Amazonas.

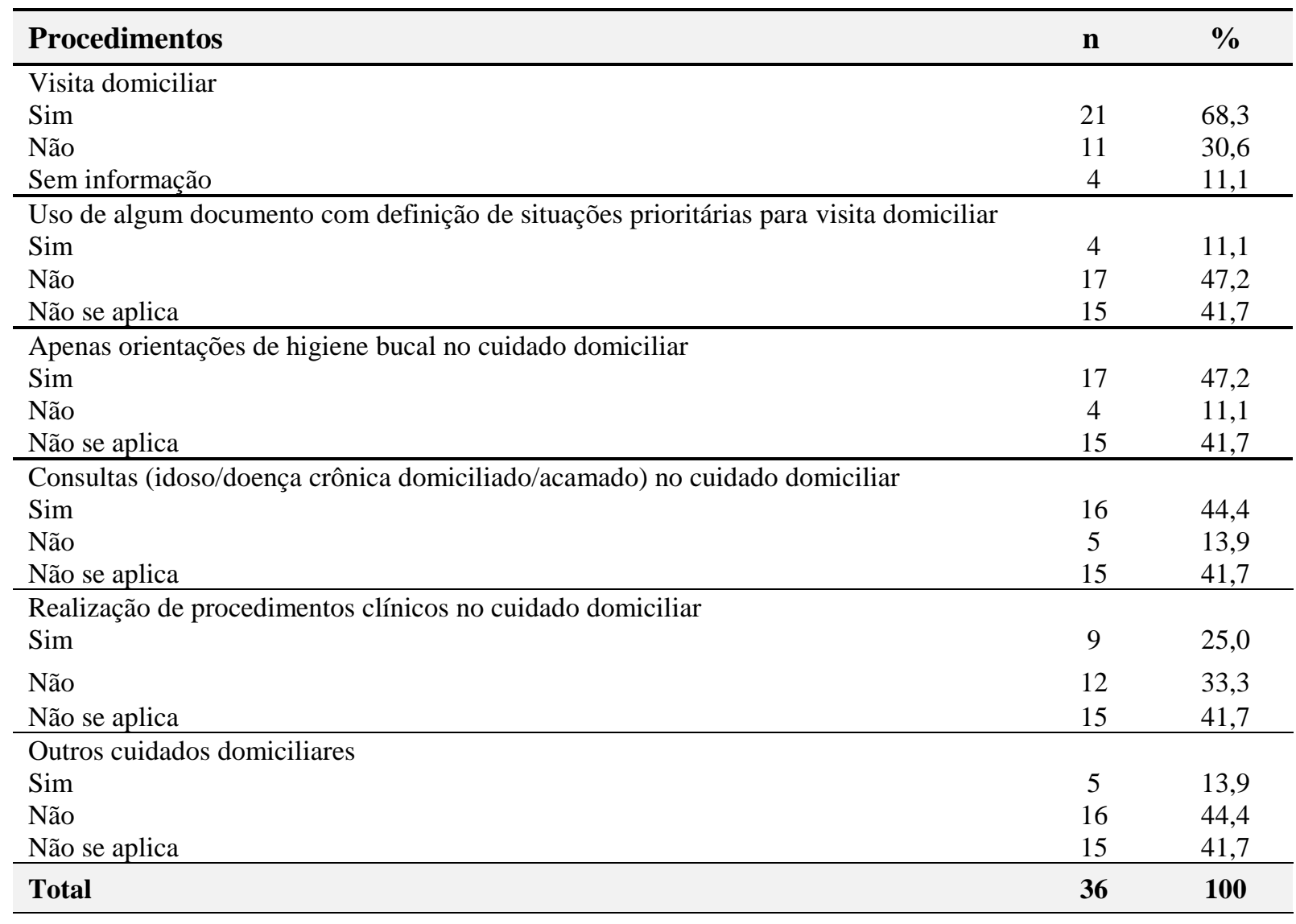

Fonte: PMAQ, 2013. 
Tabela 2- Informações em valores absolutos e percentuais sobre a Biópsia e Câncer de boca na ESB nos municípios do Baixo Amazonas.

\begin{tabular}{lcc}
\hline Procedimentos & $\mathbf{n}$ & $\mathbf{\%}$ \\
\hline O CD realiza coleta do material da biopsia & 4 & 11,1 \\
Sim & 28 & 77,8 \\
Não & 4 & 11,1 \\
Sem informação & & \\
\hline Agendamento & 2 & 5,6 \\
No mesmo momento & 2 & 5,6 \\
Até uma semana & 32 & 88,8 \\
Não se aplica & & \\
\hline Prazo para recebimento do laudo do patologista partir da coleta & 2,8 \\
Até 15 dias & 1 & 2,8 \\
Entre 15 e 30 dias & 1 & 5,6 \\
Entre 30 e 60 dias & 2 & 88,9 \\
Não se aplica & 32 & 8,3 \\
\hline ESB possui registro de casos suspeitos /confirmados de CA de boca & & 40,6 \\
Sim & 3 & 11,1 \\
Não & 13 \\
Sem informação & 4 & \\
\hline ESB realiza ações de prevenção de CA & & 63,9 \\
Sim & 23 & 25,0 \\
Não & 9 & 11,1 \\
Sem informação & 4 & $\mathbf{1 0 0}$ \\
\hline Total & $\mathbf{3 6}$ \\
\hline
\end{tabular}

Fonte: PMAQ, 2013. 
Tabela 3- Procedimentos clínicos realizados pela ESB nos municípios do Baixo Amazonas.

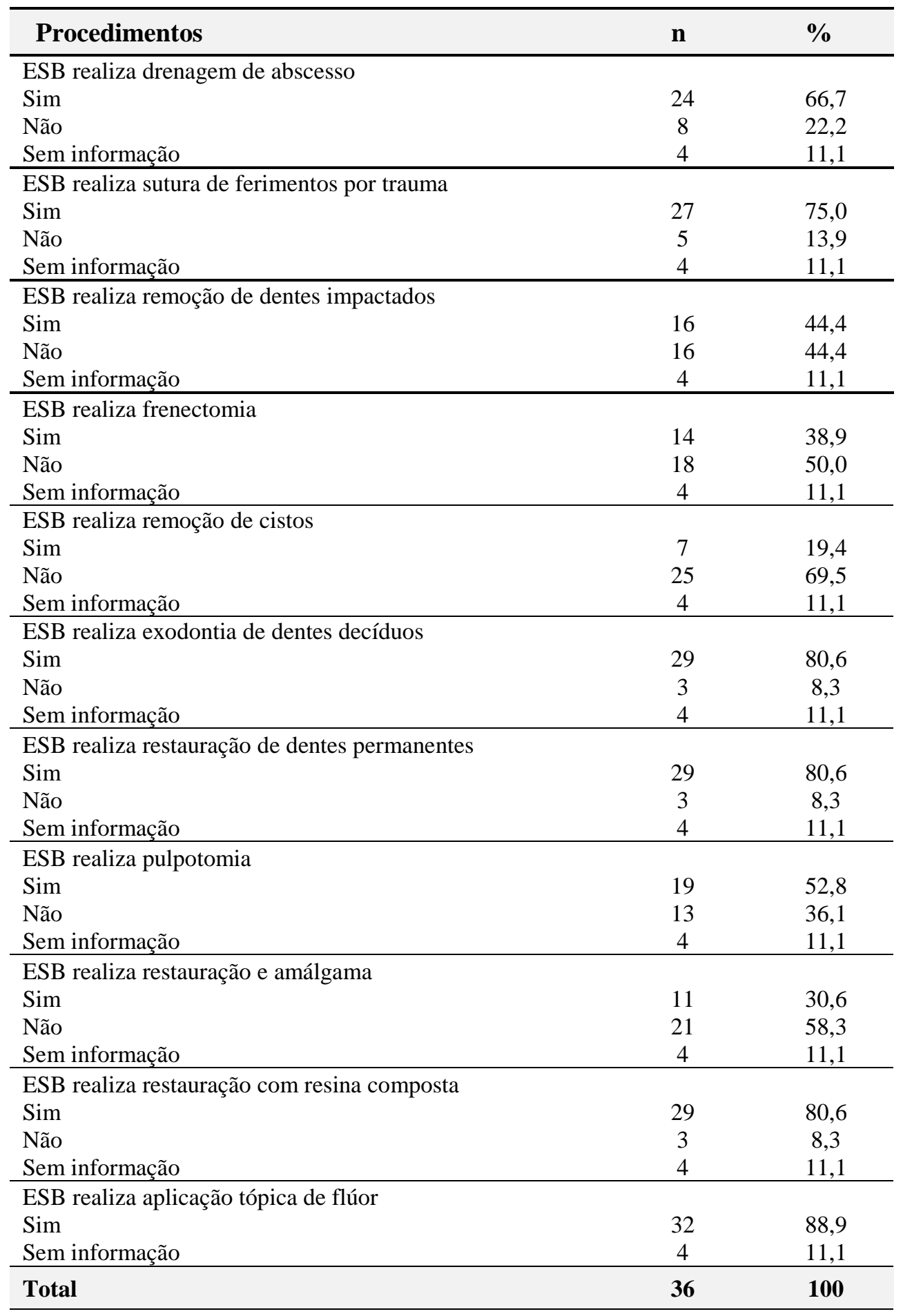

Fonte: PMAQ, 2013. 
Tabela 4- Atendimentos a gestantes e escolares realizados pela ESB nos municípios do Baixo Amazonas.

\begin{tabular}{|c|c|c|}
\hline Procedimentos & $\mathbf{n}$ & $\%$ \\
\hline \multicolumn{3}{|c|}{ ESB realiza acompanhamento das gestantes por meio de consultas } \\
\hline Sim & 27 & 75,0 \\
\hline Não & 5 & 13,9 \\
\hline Sem informação & 4 & 11,1 \\
\hline \multicolumn{3}{|c|}{ ESB realiza atendimento em crianças de até 5 anos de idade } \\
\hline Sim & 31 & 86,1 \\
\hline Não & 1 & 2,8 \\
\hline Sem informação & 4 & 11,1 \\
\hline \multicolumn{3}{|c|}{ ESB realiza atividades na escola/creche (PSE) } \\
\hline Sim & 26 & 72,2 \\
\hline Não & 6 & 16,7 \\
\hline Sem informação & 4 & 11,1 \\
\hline \multicolumn{3}{|c|}{ ESB possui registro das atividades realizadas na escola/creche. } \\
\hline Sim & 19 & 52,8 \\
\hline Não & 7 & 19,4 \\
\hline Sem informação & 10 & 27,4 \\
\hline \multicolumn{3}{|c|}{$\begin{array}{l}\text { ESB realiza possui levantamento do número de escolares com necessidade } \\
\text { de tratamento }\end{array}$} \\
\hline Sim & 13 & 36,1 \\
\hline Não & 6 & 16,7 \\
\hline Sem informação & 17 & 47,2 \\
\hline Total & 36 & 100 \\
\hline
\end{tabular}

Fonte: PMAQ, 2013. 